\title{
Prime Editing Efficiency and Fidelity are Enhanced in the Absence of
}

\section{Mismatch Repair}

4 Ferreira da Silva $\mathrm{J}^{1,2 \#}$, Oliveira $\mathrm{GP}^{1 \#}$, Arasa-Verge $\mathrm{EA}^{1}$, Moretton $\mathrm{A}^{1,2}$, Timelthaler $\mathrm{G}^{1}$, Jiricny $\mathrm{J}^{4,5}$, Loizou $\mathrm{JI}^{1,2^{*}}$

${ }^{1}$ Institute of Cancer Research, Department of Medicine I, Comprehensive Cancer Centre,

8 Medical University of Vienna, Borschkegasse 8a, 1090 Vienna, Austria

$9{ }^{2}$ CeMM Research Center for Molecular Medicine of the Austrian Academy of Sciences,

10 Vienna, Austria

$11{ }^{3}$ Institute of Life Sciences, University of Zurich, Winterthurerstrasse 190, 8057 Zurich,

12 Switzerland

$13{ }^{4}$ Institute of Biochemistry of the ETH Zurich, Otto-Stern-Weg 3, 8093 Zurich, Switzerland.

14 "Equal contribution

$15 *$ Correspondence

Correspondence: joanna.Ioizou@meduniwien.ac.at (JIL)

\section{Running title}

Enhancement of Prime Editing Efficiency and Fidelity by Ablation of Mismatch Repair

\section{Keywords}

23 Prime editing; mismatch repair; genome engineering; DNA repair

\section{Abstract}

Prime editing is a powerful genome engineering approach that enables the introduction of base substitutions, insertions and deletions, into any given genomic locus. But prime editing, at even the same locus, can exhibit wildly different efficiencies in various cell backgrounds. It is unclear what determines these variations in efficiencies in a given cellular context. Through a focused genetic screen targeting DNA repair factors, we show that the efficiency of prime editing is attenuated by the mismatch repair pathway. The accumulation of the mismatch repair protein MLH1 at sites of prime editing, indicates that mismatch repair acts at these regions to directly counteract the insertion of the edit. Consequently, ablation of mismatch repair yields an up to 17 -fold increase in prime editing efficiency across different human cell 
requirements for prime editing and identify that ablation of mismatch repair increases editing efficiency and fidelity.

\section{Introduction}

40 Genome editing technologies, using CRISPR-Cas, are powerful approaches that can be used for functional genomics, as well as for therapeutic applications (Jinek et al., 2012). Their optimal implementation, however, relies on a thorough understanding of the DNA metabolic pathways and mechanisms that are responsible for editing outcomes, as well as the activity of these pathways in different tissue contexts (Richardson et al., 2018; Yeh et al., 2019; Ferreira da Silva et al., 2021; Hussmann et al., 2021). Amongst the toolbox of Cas9 proteins that are used for genome engineering are Cas 9 nucleases that can be directed to a specific region of the genome by a single-guide RNA (sgRNA). Following the generation of a DNA double-strand break (DSB), the endogenous cellular DNA end-joining pathways can repair the lesion in an error prone manner, leading to insertions and deletions (indels) that culminate in loss-of-function alleles (Bothmer et al., 2017). This approach can be further adapted to include a donor template, either single-stranded or double-stranded, containing a desired edit. Here, following the generation of a DNA DSB, homology directed repair (HDR) mediates the insertion of the desired template, thus editing the genome. This approach can be used to introduce or correct mutations with high precision. However, since HDR is an inefficient mechanism, depends on potentially deleterious DSBs and requires cell division, other approaches have been developed.

Amongst these are base editors (BE) which use nucleobase modification chemistry to efficiently and precisely incorporate single nucleotide variants into the genome of living cells (Komor et al., 2016; Gaudelli et al., 2017; Gu et al., 2021). BEs are however limited to singlebase substitutions. Thus, more recently, prime editing (PE) was developed as a highly versatile genome editing approach that allows for targeted insertions, deletions and all possible combinations of base substitutions in a precise manner, independent on cell division (Anzalone et al., 2019). PE utilises a fusion of a Cas9(H840A) nickase (Jinek et al., 2012) and reverse transcriptase (RT) that is targeted to a precise genomic region by a PE guide RNA (pegRNA). The pegRNA encodes, in its 3' extension region, the genetic information that is copied to the targeted genomic locus by the RT. The installation of the desired edit is dependent on the resolution of a DNA intermediate that contains redundant single-stranded DNA (ssDNA) flaps. Preferential 5' flap excision and 3' flap ligation drives the integration of the edit, resulting in a DNA heteroduplex containing one edited- and one non-edited strand (PE2). The editing outcome depends on the resolution of this heteroduplex. Utilising a sgRNA 
(PE3), or afterwards (PE3b), has been shown to increase PE efficiencies (Anzalone et al., 2019).

A notable feature of prime editing is its highly variable rates of efficiency across different genetic backgrounds, even within the same genomic region and using the same pegRNA (Anzalone et al., 2019). To address whether this could be explained by different DNA repair capacities, we performed a targeted genetic screen aimed at identifying DNA repair factors involved in PE. Here, we uncover an unexpected inhibitory role for the mismatch repair (MMR) pathway in PE and show that MMR localises to sites of PE to counteract edit installation. Thus, MMR depletion increases PE efficiency and fidelity across different edit sites, types and cell lines.

\section{Results}

A targeted genetic screen identifies the mismatch repair pathway as inhibitory for prime editing

To investigate the DNA repair requirements for $\mathrm{PE}$, we conducted a targeted genetic screen, utilising a collection of isogenic knockout cell lines in the human near-haploid cell line HAP1 (Supplementary Table 1). The 32 targeted genes were selected to represent divergent functions within all known human DNA repair pathways. The library thus provided a comprehensive coverage of the DNA damage response. The cell lines received the PE machinery, including the Cas9(H840)-RT and a pegRNA encoding a 5-base pair (bp) deletion in the HEK3 locus. Editing efficiency was then determined by amplicon sequencing of the genomic locus.

Wild-type HAP1 cells were remarkably inefficient at prime editing ( $<1 \%$ alleles edited). In contrast, isogenic HAP1 cell lines mutated at the MLH1, PSM2, MSH2, EXO1 and MSH3 loci displayed higher levels of PE (ranging from 2.3 to 7.9-fold) (Figure 1A). Deficiencies in other DNA repair pathways had little impact on the efficacy of prime editing. This finding clearly indicates that MMR plays an inhibitory role in PE. Of all MMR genes targeted in the screen, only the loss of MSH6 failed to increase PE efficiency.

The MMR pathway evolved to correct base/base mispairs and indels arising in DNA during replication. Base/base mismatches and small indels of 1-3 nucleotides are recognised by the heterodimer $\mathrm{MSH} 2-\mathrm{MSH}$, whereas indels are generally recognised by the MSH2-MSH3 heterodimer (Drummond et al., 1995; Palombo et al., 1995, 1996; Acharya et al., 1996; Gradia et al., 1997). Detection of the lesion is followed by an ATP-dependent conformational change of these complexes and recruitment of the MLH1-PMS2 (Li and Modrich, 1995) or MLH1- 
110 MLH3 (Lipkin et al., 2000) heterodimers. Assembly of this complex together with RFC and 111 PCNA (Pluciennik et al., 2010) activates cryptic endonucleases of the PMS or MLH3 proteins, 112 which then introduce DNA single-strand breaks (SSBs) into the nascent DNA strand in the

113 vicinity of the mismatch. These SSBs act as entry points for EXO1, which degrades the 114 nascent strand up to, and some distance past, the misincorporated nucleotide(s) (Kadyrov et 115 al., 2006). The resulting gap is filled-in by polymerase $\delta$ and the remaining nick is ligated by 116 DNA ligase I (Stojic et al., 2004; lyer et al., 2006; Fishel, 2015). The edit introduced in our 117 screen, a $5 \mathrm{bp}$ deletion, is a preferential target of the MSH2-MSH3 heterodimer, but not the 118 MSH2-MSH6 heterodimer (Palombo et al., 1996), thus explaining the lack of an effect on 119 editing upon loss of MSH6 (Figure 1A). This result highlights the highly specialised nature of 120 the DNA damage response and shows that different edits might be addressed by distinct 121 factors.

A

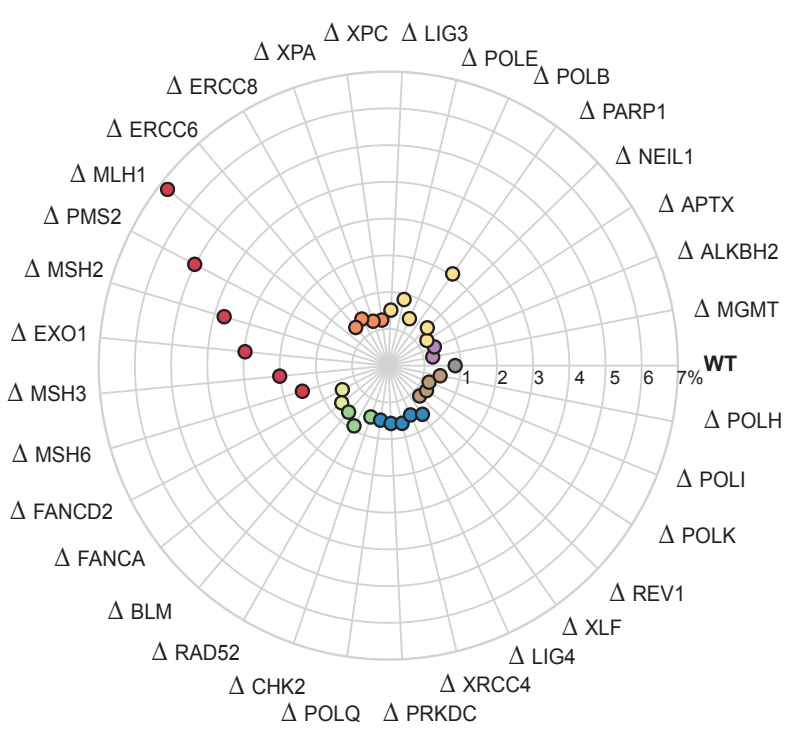

Figure 1
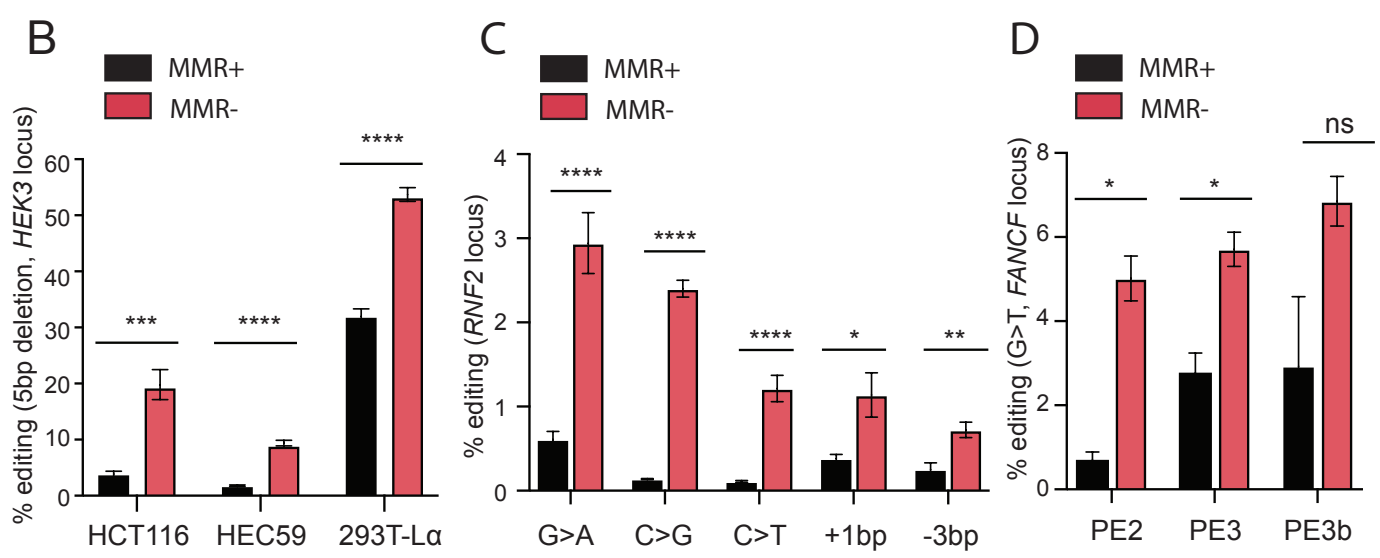

123 Figure 1: Mismatch repair inhibits prime editing in human cells. A) Genetic screen in a 124 panel of 32 HAP1 isogenic knockout cell lines covering different DNA damage repair 
pathways, as well as their wild-type (WT) counterpart, showing the efficiency of installation of a 5 base pair (bp) deletion in the HEK3 locus, using PE2. Values correspond to editing efficiency, measured by amplicon sequencing analysis in two independent biological replicates, with two technical replicates each. Each radial line represents an increment of $1 \%$. B) PE efficiency of a 5 bp deletion in the HEK3 locus in different mismatch repair-deficient human cell lines, along with their respective complemented counterparts. Values correspond to editing efficiency, measured by amplicon sequencing analysis in three independent biological replicates, with two technical replicates each. C) PE efficiency of different types of edits (RNF2 locus) in the HEC59 cell line complemented with chromosome 2 (MMR proficient; MMR+) and HEC59 cell line (MMR deficient; MMR-). Values correspond to editing efficiency, measured by amplicon sequencing analysis in three independent biological replicates, with two technical replicates each. D) Comparison of different types of PE efficiency (PE2, PE3 and PE3b) upon installation of a G>T substitution in the FANCF locus, in HEC59 cells complemented with chromosome 2 (MMR+) and HEC59 cells (MMR-). Values correspond to editing efficiency, measured by amplicon sequencing analysis in three independent biological replicates, with two technical replicates each. Ns, p-value non-significant; ${ }^{*}$, p-value $<0.05$; ${ }^{*}$, $\mathrm{p}$-value $<0.01 ;{ }^{* * *}, \mathrm{p}$-value $<0.001 ;{ }^{* * * *}$, p-value $<0.0001$.

\section{Mismatch repair hinders PE2 and PE3 across several human cell lines, genomic loci and edits}

To further explore the inhibitory role of MMR in PE, we measured editing efficiency and fidelity of the HEK3 locus in a panel of MMR-deficient human cell lines, alongside their complemented counterparts. We used the colorectal cancer line HCT116, which is deficient for both MSH3 and MLH1, along with an HCT116 MMR-proficient cell line complemented with both chromosomes 5 and 3 (Supplementary Figure 1A) (Koi et al., 1994; Haugen et al., 2008). Additionally, the endometrial adenocarcinoma cell line HEC59, which is deficient for MSH2, was used together with its MMR-proficient counterpart that was complemented with chromosome 2 (Supplementary Figure 1B) (Umar et al., 1997). Finally, we used a doxycycline-inducible model of MLH1 deficiency in the embryonic kidney cell line HEK293T (293T-La) (Supplementary Figure 1C) (Cejka et al., 2003). All cell lines showed significantly increased PE2 editing (1.7 to 5-fold) when MMR was ablated, compared to their MMRproficient counterparts (Figure 1B). Importantly, even though PE efficiencies were increased by MMR deficiency, this did not come at the expense of higher indel frequencies at the amplicon region (Supplementary Figure 1D). Indeed, we observed that loss of MMR prevented unwanted indels at the HEK3 locus in the 293T-La cell line (Supplementary Figure 1D).

To investigate further the substrates of MMR in PE, we measured the editing efficiencies of a transition $(\mathrm{G}>\mathrm{A})$, two transversions $(\mathrm{C}>\mathrm{G}$ and $\mathrm{C}>\mathrm{T})$, a 1 bp insertion and a 3 bp deletion, all within a different endogenous locus. We found that active MMR significantly diminished the efficiency of all these edits in HEC59 cells (ranging from 2.8 to 17-fold) (Figure 1C). To test 
166 the inhibitory role of MMR on different types of PE, we measured the efficiency of PE2, PE3

167 and PE3b in the FANCF locus, via the installation of a $\mathrm{G}>\mathrm{T}$ substitution. Editing efficiency was

168 improved by loss of MMR for all types of PE, albeit to a lesser extent for PE3b (Figure 1D). In

169 summary, MMR counteracts PE efficiency across different edits and different genomic loci.

171 The mismatch repair factor MLH1 is directly recruited to sites of prime editing

172 We hypothesized that MMR could reduce PE efficiency either directly at the edit site, or

173 indirectly by other mechanisms. To address this, we determined if the MMR factor MLH1 was

174 recruited to sites of active and ongoing editing, through co-localization with Cas9(H840)-RT.

175 Cas9(H840)-RT was directed to human repetitive telomeric regions, a strategy that has proven

176 efficient for CRISPR imaging (Chen et al., 2013) (Figure 2A). With this experimental

177 approach, Cas9(H840)-RT was shown to co-localise with TRF1, a major telomere-binding

178 protein (Wang et al., 2008) (Supplementary Figures 2A-B). Similarly, endogenous MLH1 co-

179 localised with Cas9(H840)-RT-foci, in a pegRNA-dependent manner (Figures 2B-C). Based

180 on these results, we postulate that PE inhibition by MMR occurs through recruitment of MMR

181 factors to sites of editing, where the heteroduplex DNA is recognised as a mismatch and

182 removed, using the non-edited strand as a template, and thus restoring the non-edited

183 sequence of nucleotides. 


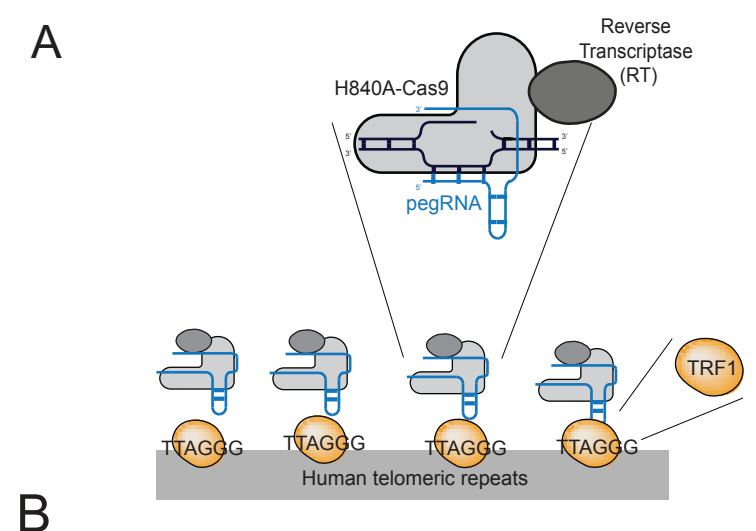

B

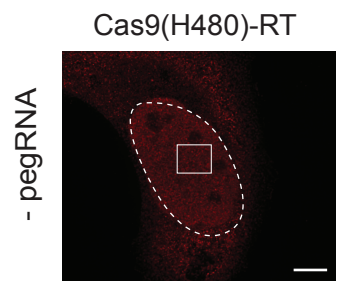

Cas9(H480)-RT

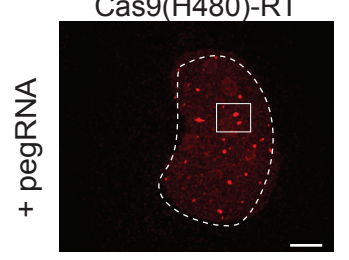

D

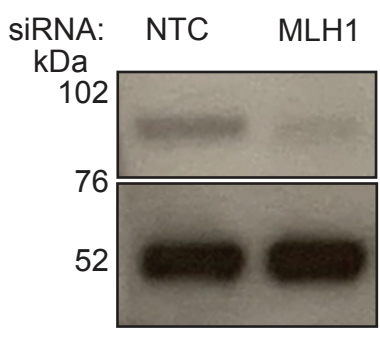

MLH1

Tubulin

$\mathrm{F}$

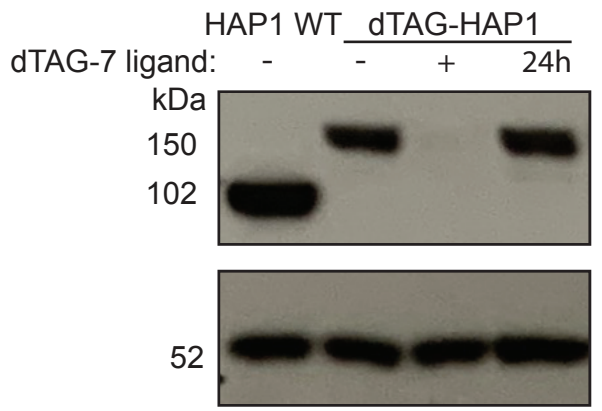

Merged

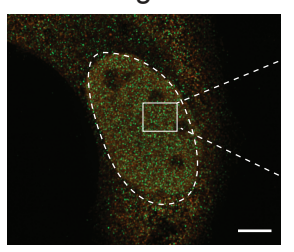

Merged

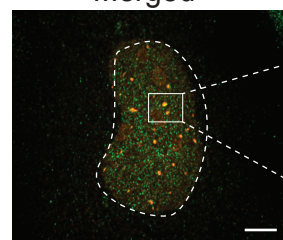

E

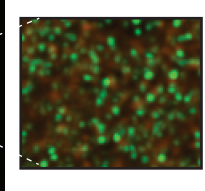

C

Figure 2
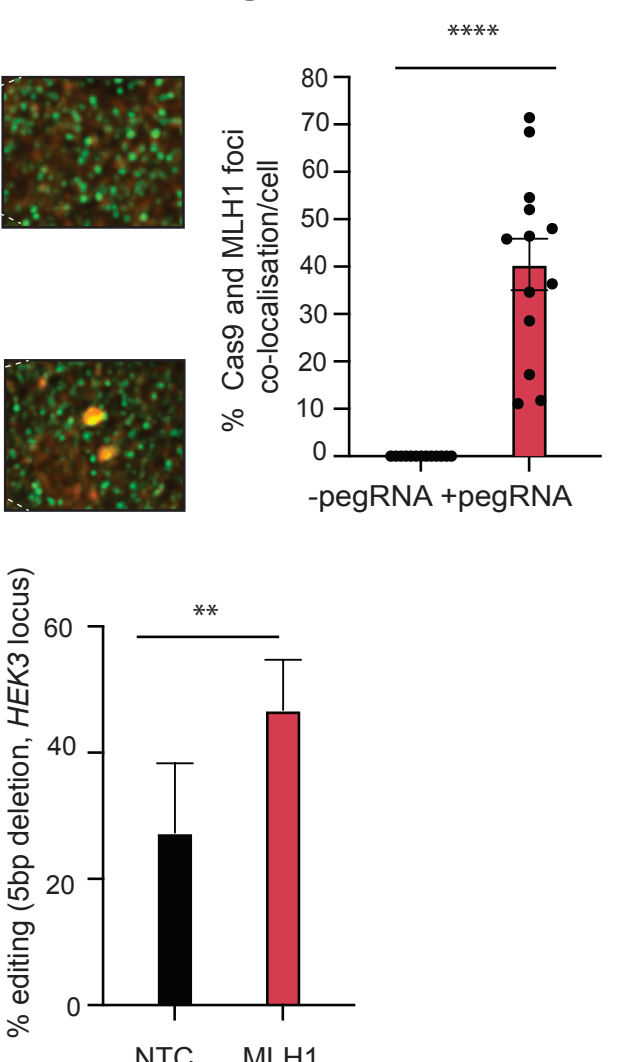

Figure 2: MLH1 is enriched at prime editing sites and its ablation can be exploited to 
189 where the telomeric protein, TRF1, also binds. B) Representative super resolution images of 190 Cas9(H490)-RT, MLH1 and both merged, in U2OS cells. Images were taken 24 hours 191 following reverse transfection of Cas9(H480)-RT and in the presence or absence of a pegRNA targeting telomeric repeats. Scale bars $=5 \mu \mathrm{m} \mathrm{C}$ ) Quantification of Cas9(H480)-RT foci that co-localise with MLH1. Values correspond to quantification of 13 cells in three independent biological replicates. D) Immunoblot for MLH1 and tubulin in HEK293 cell extracts three days post transfection with non-targeting and MLH1 siRNA pools (of four siRNAs). E) PE efficiency of a 5 bp deletion in the HEK3 locus in HEK293 cells transfected with non-targeting and MLH1 siRNA pools, two days prior to PE vector delivery. Values correspond to editing efficiency, measured by Sanger sequencing and analysed by TIDE analysis (Brinkman et al., 2014), in three independent biological replicates, with two technical replicates each. F) Immunoblot for MLH1 and tubulin in HAP1 cell extracts. Expression of dTAG-MLH1 is abrogated upon 1-hour treatment with 500nM dTAG-7 ligand ('+' condition). Recovery of dTAG-MLH1 expression is visible 24 hours after ligand removal ('24h' condition) G) PE efficiency of a 5 bp deletion in the HEK3 locus in HAP1 dTAG-MLH1 cells in the presence and absence of $500 \mathrm{nM}$ dTAG-7. Values correspond to editing efficiency, measured by Sanger sequencing and analysed by TIDE analysis (Brinkman et al., 2014), in three independent biological replicates, with two technical replicates each. Ns, $p$-value non-significant; ${ }^{*}$, $p$-value $<0.05 ;{ }^{* *}, p$-value $<0.01$; ${ }^{* * * *}$, p-value $<0.0001$

\section{Mismatch repair inhibition can be exploited to increase prime editing efficiency}

211 We next sought to inhibit MMR as a strategy to improve PE efficiency. Since MMR loss leads

212 to increased mutational burden and genome instability (Jiricny, 2006), long-term inhibition of

213 MMR is not desirable. Thus, to achieve transient MMR ablation, we depleted MLH1 in HEK293

214 cells with a pool of siRNAs (Figure 2D), and subsequently showed that this effectively increased PE efficiency in the HEK3 locus (Figure 2E).

217 An alternative way to achieve transient loss of function is through targeted protein degradation.

218 The degradation tag (dTAG) system has proved to be an efficient strategy for rapid and 219 transient ligand-induced targeted protein degradation (Winter et al., 2015). Using CRISPR220 mediated knock-in, we introduced the dTAG system into the MLH1 locus of HAP1 cells which 221 allowed for the targeted degradation of MLH1, after ligand treatment (Figure 2F). Upon MLH1 degradation, the efficiency of PE was increased by 2-fold (Figure 2G). Taken together, these results indicate that transient inhibition of MMR is a promising strategy to increase the efficiency of PE.

\section{Discussion}

227 Here, we describe an inhibitory role of the MMR pathway during PE, across different human 228 cell lines, genomic loci and types of edits, thus challenging the current view that this is a 229 pathway that is required for edit installation (Petri et al., 2021). We propose that MMR proteins 230 recognise the heteroduplex DNA, containing the desired edit introduced by the pegRNA, as a 231 mismatch. The mismatch is promptly removed, and the non-edited strand is used as a 
232 template to restore the original sequence of nucleotides. Hence, suppression of MMR 233 increases PE efficiency.

235 Our findings suggest that the improvement in PE efficiencies upon loss of MMR activity does

236 not come at the cost of indel generation around the edit site, which would be an undesirable

237 outcome of editing. Much effort has gone into improving genome editing technologies through

238 both computational and experimental approaches aimed at improving editing efficiencies and 239 fidelity. While globally manipulating MMR in dividing cells would come the expense of 240 mutations, precisely interfering with MMR at the edit site might offer an approach to specifically 241 perturb this pathway only at the sites of PE. Similar approaches have proved useful for 242 improving the efficiency of HDR (Charpentier et al., 2018; Rees et al., 2019), suggesting that 243 their development for PE might also be valuable.

245 The highly specialised nature of the DNA damage response suggests that different edits might 246 be dealt with by different pathways, and even different factors within the same pathway. Our 247 data on the lack of involvement of MSH6 in the repair of a 5bp deletion supports this hypothesis. While it is known that MMR functions to repair base substitutions and small indels, it remains to be seen what the size limitation of an edit is to be removed by this pathway, when generated by PE. Future directions might provide a deeper understanding of the specificity and size of MMR substrates in PE to determine if perturbing MMR could be used to enhance the insertion of small tags such as histidine or hemagglutinin tags.

254 Overall, our data shed new light on the DNA repair mechanisms of a new and highly promising genome editing technology. This knowledge can be applied in further development of prime editors, as well as in the design of therapeutic strategies. Finally, taking into consideration the expression levels of MMR factors in particular tissue or cell types might predict PE efficiencies.

\section{Methods}

\section{Plasmids and oligos}

262 DNA oligos were obtained from Integrated DNA Technologies (IDT) unless otherwise noted.

263 All plasmids used for prime editing experiments were obtained from Addgene (pCMV-PE2 264 no.132775, pU6-pegRNA-GG-acceptor no.132777). PegRNAs were cloned using Bsal 265 Golden Gate assembly (NEB), as described previously (Anzalone et al., 2019). The pegRNA 266 targeting telomeres included a stem loop extension as described in. SgRNAs utilized in PE3 267 and PE3b experiments were cloned in the plenti-sgRNA puro vector (Addgene no. 104990), 
using BsmBI Golden Gate assembly (NEB), following the manufacturer's instructions. The pegRNA targeting telomeres included a stem loop extension as described in (Chen et al., 2013). Sequences of sgRNA and pegRNA constructs are listed in Supplementary Table 2. All plasmids for mammalian cell experiments were purified using the Plasmid Plus Midi Kit (Qiagen) or the Spin Miniprep Kit (Qiagen), both including endotoxin removal steps.

\section{Mammalian cell culture}

All cells were grown at $3 \%$ oxygen at $37^{\circ} \mathrm{C}$ and routinely checked for possible mycoplasma contamination. Human HAP1 cells were obtained from Horizon Discovery and were grown in Iscove's Modified Dulbecco's Medium (IMDM) (Gibco), containing L-glutamine and 25nM HEPES and supplemented with 10\% Fetal Bovine Serum (FBS) (Gibco) and 1\% Penincillin/Streptomycin (P/S) (Sigma-Aldrich). U2OS cells were purchased from ATCC cell repository and cultured in DMEM (Gibco), supplemented with 10\% FBS and 1\% P/S. HEC59, wild-type and complemented with chromosome 2, were provided by Josef Jiricny (ETH Zurich, Switzerland) and cultured in F12 DMEM with 10\% FBS and 1\% P/S. HEC59 complemented cells were cultured with $400 \mu \mathrm{g} / \mathrm{mL}$ of geneticin (G418, Gibco). HCT116 cells, wild-type and complemented with both chromosomes 3 and 5, were provided by Josef Jiricny (ETH Zurich, Switzerland) and cultured with McCoy's 5A medium (Gibco), with 10\% FBS and 1\% P/S. HCT116 cells complemented with chromosomes 3 and 5 were cultured with $400 \mu \mathrm{g} / \mathrm{mL}$ geneticin (G418, Gibco) and $6 \mathrm{ug} / \mathrm{mL}$ blasticidine (Invivogen). 293T-La were provided by Josef Jiricny (ETH Zurich, Switzerland) and cultured in DMEM medium (Gibco) supplemented with $10 \%$ FBS or Tet-system approved FBS (Takara Bio), 1\% P/S, $100 \mu \mathrm{g} / \mathrm{mL}$ zeocin (Gibco) and $300 \mu \mathrm{g} / \mathrm{mL}$ hygromycin (Gibco). 293T-La were grown in doxycycline $(1 \mu \mathrm{g} / \mathrm{mL})$ for 7 days before any experiment, to completely deplete MLH1 expression. Doxycycline was replenished in the medium every 2 days.

\section{Focused DNA repair genetic screen}

CRISPR-Cas9 knockouts of DNA repair genes were generated in collaboration with Horizon Genomics. Sequences of sgRNAs were designed by Horizon Genomics or with the use of http://chopchop.cbu.uib.no/. sgRNA sequences and frameshift mutations can be found in

\section{Supplementary Table 1.}

300 For the genetic screen, 80,000 cells were seeded in technical duplicates in 12-well plates. 301 Cells were transfected the day after with 636ng of pCMV-PE2 and 159ng of the HEK3 pegRNA inducing a 5bp deletion, per well. 1.6uL Lipofectamine 2000 (ThermoFisher Scientific) were used per well, following the manufacturer's instructions. A separate transfection control was 
reagents was removed $16 \mathrm{~h}$ post-transfection. Transfection efficiency was measured $48 \mathrm{~h}$ after transfection, by determining the percentage of GFP+ cells by flow cytometry. Genomic DNA was harvested 96h post-transfection, using the QUIAmp DNA Blood Mini kit (Quiagen), following the manufacturer's instructions.

\section{Transfection and genomic DNA preparation of mismatch repair-deficient human cell}

311 lines

312 HEC59, HCT116 and HEK293Ta were seeded in 48-well plates in duplicates $(50,000$

313 cells/well). Transfections were performed the next day, using $1 \mu \mathrm{L}$ Lipofectamine 2000

314 (ThermoFisher Scientific) per well, following the manufacturer's instructions. Cells were

315 transfected with $320 \mathrm{ng}$ of the pCMV-PE2 vector, $80 \mathrm{ng}$ of the respective pegRNA and, for

316 PE3 and PE3b, $33.2 \mathrm{ng}$ of the nicking sgRNA, per well. A transfection control was performed

317 in parallel, by transfecting 400 ng per well of the pmaxGFP ${ }^{\mathrm{TM}}$ vector (Lonza).

319 Genomic DNA was extracted 96 hours after transfection, by removing the medium, 320 resuspending the cells in a lysis solution (100 $\mu \mathrm{L}$ DirectPCR Lysis Reagent (Cell) (Viagen

321 Biotech), $76 \mu \mathrm{L}$ of water and $4 \mu \mathrm{L}$ Proteinase $\mathrm{K}$ ) and incubating 45 minutes at $55^{\circ} \mathrm{C}$ and 45 322 minutes at $85^{\circ} \mathrm{C}$.

\section{High-throughput DNA sequencing of genomic samples}

325 Genomic sites of interest were amplified from genomic DNA samples and sequenced on an 326 Illumina Miseq or NextSeq, depending on the number of pooled samples. Amplification primers 327 containing Illumina forward and reverse primers (Supplementary Table 2) were used for a 328 first round of PCR (PCR1) to amplify the genomic region of interest. A mixture of staggered 329 forward primers was used to create complexity. PCR1 reactions were performed in a final 330 volume of $25 \mu \mathrm{L}$, using $0.5 \mu \mathrm{M}$ of each forward and reverse primers, $1 \mu \mathrm{L}$ genomic DNA and $331 \quad 12.5 \mu \mathrm{L}$ of Phusion U Multiplex PCR 2x Master Mix (ThermoFisher Scientific). PCR1 was carried as following: $98^{\circ} \mathrm{C} 2 \mathrm{~min}, 30$ cycles $\left[98^{\circ} \mathrm{C} 10 \mathrm{~s}, 61^{\circ} \mathrm{C} 20 \mathrm{~s}, 72^{\circ} \mathrm{C} 30 \mathrm{~s}\right.$ ], followed by a

334 to each sample in a secondary PCR reaction (PCR2). PCR2 was performed in a final volume 335 of $25 \mu \mathrm{L}$, using $0.5 \mu \mathrm{M}$ of each unique forward and reverse Illumina barcoding primer pair, 1 $336 \mu \mathrm{L}$ of unpurified PCR1 reaction and $12.5 \mu \mathrm{L}$ of of Phusion U Multiplex PCR 2x Master Mix. 337 PCR2 was carried as following: $98^{\circ} \mathrm{C} 2 \mathrm{~min}, 12$ cycles $\left[98^{\circ} \mathrm{C} 10 \mathrm{~s}, 61^{\circ} \mathrm{C} 20 \mathrm{~s}, 72{ }^{\circ} \mathrm{C} 30 \mathrm{~s}\right.$ ], 338 followed by a final extension of $72{ }^{\circ} \mathrm{C}$ for $7 \mathrm{~min}$. PCR products were analysed by 339 electrophoresis in a 1\% (w/v) agarose gel and purified using magnetic AMPure XP beads 340 (Beckman Coulter), using a ratio of beads:PCR product of 2:1. DNA concentration was 
measured by fluorometric quantification (Qubit, ThermoFisher Scientific) and sequenced on an Illumina instrument, according to manufacturer's instructions.

344 Sequencing reads were demultiplexed using MiSeq Reporter (Illumina) and alignment of 345 amplicon sequences to a reference sequence was performed using CRISPResso 2 (Clement 346 et al., 2019). CRISPResso2 was ran in standard mode and prime editing yield was calculated as: number of aligned reads containing the desired edit/total aligned reads. Percentage of indels was calculated as: number of aligned reads containing indels that are not the desired edit/ total number of aligned reads.

\section{siRNA transfections}

352 The following siRNAs from Dhamacon (used at a final concentration of 100nM) were used in

353 this study: MLH1 SMARTpool ON-TARGETplus (L-003906-00-0005) and Non-targeting 354 control SMARTpool ON-TARGETplus (D-001810-10-05). siRNA transfections in HEK293 cells were performed using Dharmafect 1 following manufacturer's instructions. siRNA delivery was performed 48 hours prior to transfection of prime editing vectors.

\section{Generation of dTAG-MLH1 HAP1 cell line}

A targeting vector encoding for the BSD-dTAG sequence (amplified from pCRIS-PITChv2BSD-dTAG (BRD4) Addgene no.91792) surrounded by two 1kb-long homology arms upstream and downstream of the start codon of MLH1 was generated using Gibson assembly (NEB). An in-vitro transcribed sgRNA targeting the region spanning the start codon of MLH1 was generated as previously described (Richardson et al., 2018). Cas9 protein (IDT) together with the targeting vector and in-vitro transcribed sgRNA were nucleofected into 200,000 haploid cells in 16-well strips, using a 4D Nucleofector (Lonza) and the program DS-118. Three days after nucleofection, $10 \mu \mathrm{g} / \mathrm{ml}$ blasticidin (Invivogen) were added to the culture medium for one week after which single and haploid clones were sorted into 96-well plates. Clonal haploid populations were grown and validated for correct homology-directed repair by LR-PCR and immuno blot analysis. dTAG-7 (R\&D Systems), at the final concentration of 500 $\mathrm{nM}$, was used to test target degradation in the generated clones and all further targeted protein degradation experiments.

\section{Immunoblotting}

375 Cell extracts were prepared in RIPA lysis buffer (NEB) supplemented with protease inhibitors

376 (Sigma) and phosphatase inhibitors (Sigma, NEB). Immunoblots were performed using 377 standard procedures. Protein samples were separated by sodium dodecyl sulfate- 
polyacrylamide gel electrophoresis (SDS-PAGE) (3-8\% gradient gels, Invitrogen) and subsequently transferred onto nitrocellulose membranes. Primary antibodies for MLH1 (554073, BD Pharmigen), MSH2 (ab52266, Abcam), MSH3 (ab69619, Abcam), Tubulin (3873, Cell Signaling) and B-Actin (A5060, Sigma) were used at 1:1,000. Secondary antibodies were used at 1:5,000 (HRP-conjugated goat anti-mouse or anti-rabbit IgG from Jackson Immunochemicals). Immunoblots were imaged using a Curix 60 (AGFA) table-top processor.

\section{Immunofluorescence}

U2OS cells were reverse transfected using PEI together with PCMV-PE2 in the presence and absence of pegRNA targeting telomeres. 50.000 cells were seeded per well of $\mu$-Slide 8 well (Ibidi) chambered coverslip plates. Pre-extraction was performed using $0.1 \%$ Tween in PBS 24 hours after reverse transfection. Cells were then fixed with $4 \%$ para-formaldehyde and fixed cells were processed for immunofluorescence using the following antibodies: anti-Cas9 (Cell Signalling, 14697), anti-TRF1 (Abcam, ab1423), anti-MLH1 (ThermoFisher, A300-015A), antiMSH2 (Bethyl, A300-452A). Primary antibodies were diluted 1:500 and secondary antibodies (Alexa Fluor ${ }^{\circledR} 568$ goat anti-mouse and Alexa Fluor ${ }^{\circledR} 488$ goat anti-rabbit, LifeTechnologies) were diluted 1:2000.

\section{Imaging}

16-bit fluorescence images were acquired using an Olympus IXplore spinning disk confocal microscope (equipped with the Yokogawa CSU-W1 with $50 \mu \mathrm{m}$ pinhole disk and a Hamamatsu ORCA Fusion CMOS camera). A 60X oil immersion objective (NA 1,42) in combination with a $3,2 \mathrm{X}$ magnification lens (equaling 192X total magnification) was used for superresolution imaging of fixed cells and $z$-stacks with a $0,24 \mu \mathrm{m}$ slice interval were acquired. These $z$-stacks were then processed using the Olympus 3D deconvolution software (constrained iterative deconvolution, using automatic background removal and noise reduction, filter using advanced maximum likelihood algorithm and 5 iterations). Finally "maximum-z" projection images of the deconvoluted z-stacks were generated. For further data analysis, the ImageJ (NIH) distribution FIJI was used. Nuclear foci were counted manually and at least 15 cells per condition were imaged in each experiment. Quantification of the foci was performed manually based on maximum intensity projections.

\section{References}

Acharya, S., Wilson, T., Gradia, T., Kane, M., Guerrette, S., Marsischky, G., et al. (1996). hMSH2 forms specific mispair-binding complexes with hMSH3 and hMSH6. Proc. Natl. 
Acad. Sci. U. S. A. 93, 13629-13634. doi:10.1073/PNAS.93.24.13629.

Anzalone, A. V, Randolph, P. B., Davis, J. R., Sousa, A. A., Koblan, L. W., Levy, J. M., et al. (2019). Search-and-replace genome editing without double-strand breaks or donor DNA. doi:10.1038/s41586-019-1711-4.

Bothmer, A., Phadke, T., Barrera, L. A., Margulies, C. M., Lee, C. S., Buquicchio, F., et al. (2017). Characterization of the interplay between DNA repair and CRISPR/Cas9induced DNA lesions at an endogenous locus. Nat. Commun. 8, 13905. doi:10.1038/ncomms13905.

Brinkman, E. K., Chen, T., Amendola, M., and van Steensel, B. (2014). Easy quantitative assessment of genome editing by sequence trace decomposition. Nucleic Acids Res. 42, e168-e168. doi:10.1093/NAR/GKU936.

Cejka, P., Stojic, L., Mojas, N., Russell, A. M., Heinimann, K., Cannavó, E., et al. (2003). Methylation-induced $\mathrm{G} 2 / \mathrm{M}$ arrest requires a full complement of the mismatch repair protein hMLH1. EMBO J. 22, 2245. doi:10.1093/EMBOJ/CDG216.

Charpentier, M., Khedher, A. H. Y., Menoret, S., Brion, A., Lamribet, K., Dardillac, E., et al. (2018). CtIP fusion to Cas 9 enhances transgene integration by homology-dependent repair. Nat. Commun. 9, 1-11. doi:10.1038/s41467-018-03475-7.

Chen, B., Gilbert, L., Cimini, B., Schnitzbauer, J., Zhang, W., Li, G., et al. (2013). Dynamic imaging of genomic loci in living human cells by an optimized CRISPR/Cas system. Cell 155, 1479-1491. doi:10.1016/J.CELL.2013.12.001.

Clement, K., Rees, H., Canver, M. C., Gehrke, J. M., Farouni, R., Hsu, J. Y., et al. (2019). CRISPResso2 provides accurate and rapid genome editing sequence analysis. Nat. Biotechnol. 2019373 37, 224-226. doi:10.1038/s41587-019-0032-3.

Drummond, J., Li, G., Longley, M., and Modrich, P. (1995). Isolation of an hMSH2-p160 heterodimer that restores DNA mismatch repair to tumor cells. Science 268, 1909 1912. doi:10.1126/SCIENCE.7604264.

Ferreira da Silva, J., Meyenberg, M., and Loizou, J. I. (2021). Tissue specificity of DNA repair: the CRISPR compass. Trends Genet. 0. doi:10.1016/J.TIG.2021.07.010.

Fishel, R. (2015). Mismatch repair. J. Biol. Chem. 290, 26395-26403. doi:10.1074/JBC.R115.660142.

Gaudelli, N. M., Komor, A. C., Rees, H. A., Packer, M. S., Badran, A. H., Bryson, D. I., et al. (2017). Programmable base editing of $T$ to $G C$ in genomic DNA without DNA cleavage. Nature 551, 464-471. doi:10.1038/nature24644.

Gradia, S., Acharya, S., and Fishel, R. (1997). The Human Mismatch Recognition Complex hMSH2-hMSH6 Functions as a Novel Molecular Switch. Cell 91, 995-1005. doi:10.1016/S0092-8674(00)80490-0.

Gu, S., Bodai, Z., Cowan, Q. T., and Komor, A. C. (2021). Base editors: Expanding the types 
of DNA damage products harnessed for genome editing. Gene Genome Ed. 1, 100005. doi:10.1016/J.GGEDIT.2021.100005.

Haugen, A. C., Goel, A., Yamada, K., Marra, G., Nguyen, T.-P., Nagasaka, T., et al. (2008). Genetic Instability Caused by Loss of MutS Homologue 3 in Human Colorectal Cancer. Cancer Res. 68, 8465-8472. doi:10.1158/0008-5472.CAN-08-0002.

Hussmann, J. A., Ling, J., Ravisankar, P., Yan, J., Cirincione, A., Xu, A., et al. (2021). Mapping the Genetic Landscape of DNA Double-strand Break Repair. bioRxiv, 2021.06.14.448344. doi:10.1101/2021.06.14.448344.

lyer, R., Pluciennik, A., Burdett, V., and Modrich, P. (2006). DNA mismatch repair: functions and mechanisms. Chem. Rev. 106, 302-323. doi:10.1021/CR0404794.

Jinek, M., Chylinski, K., Fonfara, I., Hauer, M., Doudna, J. A., and Charpentier, E. (2012). A programmable dual-RNA-guided DNA endonuclease in adaptive bacterial immunity. Science (80-. ). 337, 816-821. doi:10.1126/science.1225829.

Jiricny, J. (2006). The multifaceted mismatch-repair system. Nat. Rev. Mol. Cell Biol. 2006 75 7, 335-346. doi:10.1038/nrm1907.

Kadyrov, F., Dzantiev, L., Constantin, N., and Modrich, P. (2006). Endonucleolytic function of MutLalpha in human mismatch repair. Cell 126, 297-308. doi:10.1016/J.CELL.2006.05.039.

Koi, M., Umar, A., Chauhan, D. P., Cherian, S. P., Carethers, J. M., Kunkei, T. A., et al. (1994). Human Chromosome 3 Corrects Mismatch Repair Deficiency and Microsatellite Instability and Reduces N-Methyl-N'-nitro-N-nitrosoguanidine Tolerance in Colon Tumor Cells with Homozygous hMLH1 Mutation. Cancer Res. 54.

Komor, A. C., Kim, Y. B., Packer, M. S., Zuris, J. A., and Liu, D. R. (2016). Programmable editing of a target base in genomic DNA without double-stranded DNA cleavage. Nature 533, 420-424. doi:10.1038/nature17946.

Li, G., and Modrich, P. (1995). Restoration of mismatch repair to nuclear extracts of H6 colorectal tumor cells by a heterodimer of human MutL homologs. Proc. Natl. Acad. Sci. U. S. A. 92, 1950-1954. doi:10.1073/PNAS.92.6.1950.

Lipkin, S., Wang, V., Jacoby, R., Banerjee-Basu, S., Baxevanis, A., Lynch, H., et al. (2000). MLH3: a DNA mismatch repair gene associated with mammalian microsatellite instability. Nat. Genet. 24, 27-35. doi:10.1038/71643.

Palombo, F., Gallinari, P., laccarino, I., Lettieri, T., Hughes, M., D'Arrigo, A., et al. (1995). GTBP, a 160-kilodalton protein essential for mismatch-binding activity in human cells. Science (80-. ). 268, 1912-1914. doi:10.1126/SCIENCE.7604265.

Palombo, F., laccarino, I., Nakajima, E., Ikejima, M., Shimada, T., and Jiricny, J. (1996). hMutS $\beta$, a heterodimer of hMSH2 and hMSH3, binds to insertion/deletion loops in DNA. Curr. Biol. 6, 1181-1184. doi:10.1016/S0960-9822(02)70685-4. 
488

489

490

491

492

493

494

495

496

497

498

499

500

501

502

503

504

505

506

507

508

509

510

511

512

513

514

515

516

517

518

519

520

521

522

523

Petri, K., Zhang, W., Ma, J., Schmidts, A., Lee, H., Horng, J. E., et al. (2021). CRISPR prime editing with ribonucleoprotein complexes in zebrafish and primary human cells. Nat. Biotechnol. 2021, 1-5. doi:10.1038/s41587-021-00901-y.

Pluciennik, A., Dzantiev, L., lyer, R. R., Constantin, N., Kadyrov, F. A., and Modrich, P. (2010). PCNA function in the activation and strand direction of MutL $\alpha$ endonuclease in mismatch repair. Proc. Natl. Acad. Sci. 107, 16066-16071. doi:10.1073/PNAS.1010662107.

Rees, H. A., Yeh, W.-H., and Liu, D. R. (2019). Development of hRad51-Cas9 nickase fusions that mediate HDR without double-stranded breaks. Nat. Commun. 201910110 , 1-12. doi:10.1038/s41467-019-09983-4.

Richardson, C. D., Kazane, K. R., Feng, S. J., Zelin, E., Bray, N. L., Schäfer, A. J., et al. (2018). CRISPR-Cas9 genome editing in human cells occurs via the Fanconi anemia pathway. Nat. Genet. doi:10.1038/s41588-018-0174-0.

Stojic, L., Brun, R., and Jiricny, J. (2004). Mismatch repair and DNA damage signalling. DNA Repair (Amst). 3, 1091-1101. doi:10.1016/J.DNAREP.2004.06.006.

Umar, A., Koi, M., Risinger, J. I., Glaab, W. E., Tindall, K. R., Kolodner, R. D., et al. (1997). Correction of Hypermutability, N-Methyl-N'-nitro-N-nitrosoguanidine Resistance, and Defective DNA Mismatch Repair by Introducing Chromosome 2 into Human Tumor Cells with Mutations in MSH2 and MSH6. Cancer Res. 57.

Wang, X., Kam, Z., Carlton, P. M., Xu, L., Sedat, J. W., and Blackburn, E. H. (2008). Rapid telomere motions in live human cells analyzed by highly time-resolved microscopy. Epigenetics Chromatin 2008 111, 1-19. doi:10.1186/1756-8935-1-4.

Winter, G. E., Buckley, D. L., Paulk, J., Roberts, J. M., Souza, A., Dhe-Paganon, S., et al. (2015). Phthalimide conjugation as a strategy for in vivo target protein degradation. Science (80-. ). 348, 1376-1381. doi:10.1126/SCIENCE.AAB1433.

Yeh, C. D., Richardson, C. D., and Corn, J. E. (2019). Advances in genome editing through control of DNA repair pathways. Nat. Cell Biol. 21(12), 1468-1478.

doi:10.1038/s41556-019-0425-z.

\section{Data availability}

All sequencing data will be deposited in the European Nucleotide Archive (EMBL-EBI).

\section{Competing Interests}

The authors declare no commercial or financial relationships that could be construed as potential conflict of interest. 


\section{Author Contributions}

526 JFdaS, GO, JJ and JIL conceptualized the study. JFdaS and JIL obtained funding. JFdaS,

527 GO, EA and AM carried out investigations. GT performed the confocal microscopy. JFdaS

528 performed analysis and visualization. JIL supervised the study. JFsaS with input from JIL

529 wrote the original draft and all authors reviewed and edited the final manuscript.

\section{$531 \quad$ Funding}

532 JFdaS is supported by a DOC fellowship from the Austrian Academy of Sciences 533 (ÖAW25035). A.M. is funded by the Austrian Science Fund, grant number P 33024 awarded 534 to J.I.L. The Loizou lab is funded by an ERC Synergy Grant (DDREAMM Grant agreement ID: 535 855741). CeMM is funded by the Austrian Academy of Sciences.

\section{Acknowledgments}

538 We would like to thank the VBCF (Vienna, Austria) for all next generation sequencing. We 539 would like to acknowledge Dr Jacob Corn (ETHZ, Zurich, Switzerland) and Dr Richard 540 Sherwood (Harvard Medical School, Boston, USA) for critically reading the manuscript. We 541 would like to thank Mr Chris Fell (Medical University of Vienna, Vienna, Austria) and Mr Marc 542 Wiedner (CeMM, Vienna, Austria) for technical support. We are thankful to Prof KJ Patel 543 (Weatherall Institute of Molecular Medicine, Oxford, UK) for providing the FANCD2 deficient 544 HAP1 cell line. The Winter Lab (CeMM, Vienna, Austria) provided valuable tools for protein 545 degradation. We are grateful to members of the Loizou lab for helpful discussions and feedback. 